\title{
A bilinear version of Holsztyński's theorem on isometries of $C(X)$-spaces
}

\author{
by \\ Antonio Moreno Galindo and \\ Ángel Rodríguez Palacios (Granada)
}

\begin{abstract}
We prove that, for a compact metric space $X$ not reduced to a point, the existence of a bilinear mapping $\diamond: C(X) \times C(X) \rightarrow C(X)$ satisfying $\|f \diamond g\|=\|f\|\|g\|$ for all $f, g \in C(X)$ is equivalent to the uncountability of $X$. This is derived from a bilinear version of Holsztyński's theorem [3] on isometries of $C(X)$-spaces, which is also proved in the paper.
\end{abstract}

1. Introduction. A celebrated theorem of W. Holsztyński [3] asserts that, if $X$ and $Z$ are compact Hausdorff topological spaces, and if $T$ : $C(X) \rightarrow C(Z)$ is a linear isometry, then there exist a closed subset $Z_{0}$ of $Z$, a continuous surjective mapping $\varphi: Z_{0} \rightarrow X$, and a norm-one element $\alpha \in C(Z)$ satisfying $|\alpha(z)|=1$ and $T(f)(z)=\alpha(z) f(\varphi(z))$ for every $(z, f) \in Z_{0} \times C(X)$. As a main result, we prove that, if $X, Y, Z$ are compact Hausdorff topological spaces, and if $\diamond: C(X) \times C(Y) \rightarrow C(Z)$ is a bilinear mapping satisfying $\|f \diamond g\|=\|f\|\|g\|$ for every $(f, g) \in C(X) \times C(Y)$, then there exist a closed subset $Z_{0}$ of $Z$, a continuous surjective mapping $\varphi: Z_{0} \rightarrow X \times Y$, and a norm-one element $\alpha \in C(Z)$ satisfying $|\alpha(z)|=1$ and

$$
(f \diamond g)(z)=\alpha(z) f\left(\pi _ { X } ( \varphi ( z ) ) g \left(\pi_{Y}(\varphi(z))\right.\right.
$$

for every $(z, f, g) \in Z_{0} \times C(X) \times C(Y)$, where $\pi_{X}: X \times Y \rightarrow X$ and $\pi_{Y}: X \times Y \rightarrow Y$ stand for the natural coordinate projections. We note that Holsztyński's original theorem follows from the new bilinear version by taking the space $Y$ reduced to a point.

We established the main result just stated while attempting to determine those compact Hausdorff topological spaces $X$ such that the Banach space $C(X)$ is "absolute-valuable". That a Banach space $E$ is absolute-valuable means that there exists a bilinear mapping $\diamond: E \times E \rightarrow E$ satisfying $\|\xi \diamond \chi\|$

2000 Mathematics Subject Classification: Primary 46E15, 46B04; Secondary 46H70.

Partially supported by Junta de Andalucía grant FQM 0199 and Projects I+D MCYT BFM2001-2335, BFM2002-01529, and BFM2002-01810. 
$=\|\xi\|\|\chi\|$ for all $\xi, \chi \in E$. The reader is referred to [1] for the present status of the theory of such spaces. We derive from the main result that, if $X$ is a compact Hausdorff topological space such that $C(X)$ is absolutevaluable, then $X$ must be either reduced to a point or not scattered. Thus we rediscover the fact, first proved in [1], that $C(X)$ is not absolute-valuable when we take $X$ equal to the one-point compactification of any infinite discrete space. We also deduce that, in the case when the compact space $X$ is metrizable and not reduced to a point, the Banach space $C(X)$ is absolute-valuable if and only if $X$ is uncountable.

2. The main result. Throughout this paper $\mathbb{K}$ will denote the field of real or complex numbers. The field $\mathbb{K}$ will remain fixed, and, for a compact Hausdorff topological space $X, C(X)$ will stand for the Banach space over $\mathbb{K}$ of all $\mathbb{K}$-valued continuous functions on $X$.

TheOREM 2.1. Let $X, Y, Z$ be compact Hausdorff topological spaces, and let $\diamond: C(X) \times C(Y) \rightarrow C(Z)$ be a bilinear mapping satisfying

$$
\|f \diamond g\|=\|f\|\|g\|
$$

for every $(f, g) \in C(X) \times C(Y)$. Then there exist a closed subset $Z_{0}$ of $Z$, a continuous surjective mapping $\varphi: Z_{0} \rightarrow X \times Y$, and a norm-one element $\alpha \in C(Z)$ satisfying $|\alpha(z)|=1$ and

$$
(f \diamond g)(z)=\alpha(z) f\left(\pi_{X}(\varphi(z))\right) g\left(\pi_{Y}(\varphi(z))\right)
$$

for every $(z, f, g) \in Z_{0} \times C(X) \times C(Y)$. Here, $\pi_{X}: X \times Y \rightarrow X$ and $\pi_{Y}: X \times Y \rightarrow Y$ denote the natural coordinate projections.

Proof. Given a compact Hausdorff topological space $K$, we denote by $\mathbf{1}_{K}$ the constant function equal to 1 on $K$, and, for $k$ in $K$, we put

$$
S_{k}:=\{f \in C(K):\|f\|=1=|f(k)|\} .
$$

Given compact Hausdorff topological spaces $K$ and $L$, an element $k$ of $K$, and a linear isometry $T: C(K) \rightarrow C(L)$, we put

$$
Q_{k}^{T}:=\left\{l \in L: T\left(S_{k}\right) \subseteq S_{l}\right\}
$$

We will apply several times the following result, proved by W. Holsztyński [3]:

(*) If $l \in Q_{k}^{T}$, then $T(f)(l)=T\left(\mathbf{1}_{K}\right)(l) f(k)$ for every $f \in C(K)$.

Now, for $(x, y) \in X \times Y$ we define

$$
Q_{x, y}:=\left\{z \in Z: S_{x} \diamond S_{y} \subseteq S_{z}\right\},
$$

and organize the proof in several steps.

SteP (i): If $(x, y) \in X \times Y$ and $(f, g) \in C(X) \times C(Y)$ are such that $f(x)=0$ or $g(y)=0$, then we have $(f \diamond g)(z)=0$ for every $z \in Q_{x, y}$. Let 
us fix $(x, y, g)$ in $X \times Y \times C(Y)$ with $g \in S_{y}$, consider the linear isometry $T: C(X) \rightarrow C(Z)$ defined by $T(h):=h \diamond g$, and note that $Q_{x, y} \subseteq Q_{x}^{T}$. Assume that $f \in C(X)$ satisfies $f(x)=0$. Then, by $(*)$, we have

$$
(f \diamond g)(z)=(T(f))(z)=0
$$

for every $z \in Q_{x}^{T}$, and in particular $(f \diamond g)(z)=0$ for every $z \in Q_{x, y}$. Now, the restriction that $g$ lies in $S_{Y}$ can be removed by keeping in mind that, since $S_{y} S_{y} \subseteq S_{y}$, the linear hull of $S_{y}$ in $C(Y)$ is a subalgebra of $C(Y)$ which is self-adjoint, contains the constants, and separates the points of $Y$, so that the Stone-Weierstrass theorem applies.

SteP (ii): If $(x, y)$ and $\left(x^{\prime}, y^{\prime}\right)$ are in $X \times Y$ with $(x, y) \neq\left(x^{\prime}, y^{\prime}\right)$, then $Q_{x, y} \cap Q_{x^{\prime}, y^{\prime}}=\emptyset$. Let $x, x^{\prime} \in X$ and $y, y^{\prime} \in Y$ be such that $x \neq x^{\prime}$, and assume that there exists $z \in Q_{x, y} \cap Q_{x^{\prime}, y^{\prime}}$. Then, taking $(f, g) \in S_{x} \times S_{y}$ with $f\left(x^{\prime}\right)=0$, we have $|(f \diamond g)(z)|=1$ (by the definition of $Q_{x, y}$ ) and $(f \diamond g)(z)=0$ (by Step (i)), a contradiction.

Step (iii): For every $(x, y) \in X \times Y$ we have $Q_{x, y} \neq \emptyset$. Let $(x, y)$ be in $X \times Y$, and let $f_{1}, \ldots, f_{n}$ and $g_{1}, \ldots, g_{n}$ be in $S_{x}$ and $S_{y}$, respectively. Putting $F:=\sum_{i=1}^{n} \overline{f_{i}(x)} f_{i}$ and $G:=\sum_{i=1}^{n} \overline{g_{i}(y)} g_{i}$, we have $|F(x)|=n=\|F\|$, $|G(y)|=n=\|G\|$, and hence $\|F \diamond G\|=n^{2}$. Therefore there exists $z \in Z$ satisfying

$$
n^{2}=|(F \diamond G)(z)|=\left|\sum_{i, j=1}^{n} \overline{f_{i}(x) g_{j}(y)}\left(f_{i} \diamond g_{j}\right)(z)\right| .
$$

This implies $\left|\left(f_{i} \diamond g_{j}\right)(z)\right|=1$ for all $i, j=1, \ldots, n$. In this way we have shown that, denoting by $\mathbb{T}$ the unit sphere of $\mathbb{K}$, the family

$$
\left\{(f \diamond g)^{-1}(\mathbb{T}):(f, g) \in S_{x} \times S_{y}\right\}
$$

has the finite intersection property. By the compactness of $Z$, we have in fact $Q_{x, y}=\bigcap_{(f, g) \in S_{x} \times S_{y}}(f \diamond g)^{-1}(\mathbb{T}) \neq \emptyset$.

Now, we consider the norm-one element $\alpha$ of $C(Z)$ defined by

$$
\alpha(z):=\left(\mathbf{1}_{X} \diamond \mathbf{1}_{Y}\right)(z) .
$$

SteP (iv): For $(x, y, z)$ in $X \times Y \times Z$ with $z \in Q_{x, y}$, we have

$$
(f \diamond g)(z)=\alpha(z) f(x) g(y)
$$

for every $(f, g) \in C(X) \times C(Y)$, and $|\alpha(z)|=1$. Since $\left(\mathbf{1}_{X}, \mathbf{1}_{Y}\right)$ belongs to $S_{x} \times S_{y}$ whenever $(x, y)$ is in $X \times Y$, it follows from the definitions of $\alpha$ and $Q_{x, y}$ that $|\alpha(z)|=1$ whenever $z$ is in $Q_{x, y}$. Now, let us fix $(x, y, f, g)$ in $X \times Y \times C(X) \times C(Y)$ with $g \in S_{y}$, and consider the linear isometries $T: C(X) \rightarrow C(Z)$ and $R: C(Y) \rightarrow C(Z)$ defined by $T(h):=h \diamond g$ and $R(h):=\mathbf{1}_{X} \diamond h$, respectively. Keeping in mind the inclusion $Q_{x, y} \subseteq Q_{x}^{T} \cap Q_{y}^{R}$, 
and applying $(*)$, for $z \in Q_{x, y}$ we derive

$$
\begin{aligned}
(f \diamond g)(z) & =T(f)(z)=T\left(\mathbf{1}_{X}\right)(z) f(x)=\left(\mathbf{1}_{X} \diamond g\right)(z) f(x)=R(g)(z) f(x) \\
& =R\left(\mathbf{1}_{Y}\right)(z) g(y) f(x)=\left(\mathbf{1}_{X} \diamond \mathbf{1}_{Y}\right)(z) f(x) g(y)=\alpha(z) f(x) g(y) .
\end{aligned}
$$

The restriction that $g$ lies in $S_{y}$ can be removed by arguing as in the conclusion of the proof of Step (i).

Now, we define $Z_{0}:=\bigcup_{(x, y) \in X \times Y} Q_{x, y}$. In view of Step (ii), for every $z \in Z_{0}$ there exists a unique $\varphi(z) \in X \times Y$ such that $z$ belongs to $Q_{\pi_{X}(\varphi(z)), \pi_{Y}(\varphi(z))}$. Moreover, by Step (iii), the mapping $\varphi: Z_{0} \rightarrow X \times Y$ defined in this way is surjective. On the other hand, by Step (iv), the norm-one element $\alpha \in C(Z)$ satisfies $|\alpha(z)|=1$ whenever $z$ lies in $Z_{0}$, and the equality

$$
(f \diamond g)(z)=\alpha(z) f\left(\pi_{X}(\varphi(z))\right) g\left(\pi_{Y}(\varphi(z))\right)
$$

holds for every $(z, f, g) \in Z_{0} \times C(X) \times C(Y)$. Thus, to conclude the proof of the theorem it is enough to establish the following.

STEP (v): $Z_{0}$ is closed in $Z$, and the mapping $\varphi: Z_{0} \rightarrow X \times Y$ is continuous. Let $A$ be a closed subset of $X \times Y$, let $z_{0}$ be in $Z \backslash \varphi^{-1}(A)$, and let $a=(x, y)$ be in $A$. Since $\varphi^{-1}(A)=\bigcup_{(x, y) \in A} Q_{x, y}$, there exists $\left(f_{a}, g_{a}\right) \in S_{x} \times S_{y}$ such that $\varepsilon_{a}:=\left(1-\left|\left(f_{a} \diamond g_{a}\right)\left(z_{0}\right)\right|\right) / 2>0$. Now, we consider the open subset $U_{a}$ of $X \times Y$ given by

$$
U_{a}:=\left\{\left(x^{\prime}, y^{\prime}\right) \in X \times Y:\left|f_{a}\left(x^{\prime}\right) g_{a}\left(y^{\prime}\right)\right|>1-\varepsilon_{a}\right\},
$$

and the disjoint open subsets $V_{a}$ and $G_{a}$ of $Z$ defined by

$$
\begin{aligned}
V_{a} & :=\left\{z \in Z:\left|\left(f_{a} \diamond g_{a}\right)(z)\right|>1-\varepsilon_{a}\right\}, \\
G_{a} & :=\left\{z \in Z:\left|\left(f_{a} \diamond g_{a}\right)(z)\right|<1-\varepsilon_{a}\right\} .
\end{aligned}
$$

We claim that $\varphi^{-1}\left(U_{a}\right) \subseteq V_{a}$. Indeed, if $z$ is in $Z_{0}$ with $\varphi(z)=\left(x^{\prime}, y^{\prime}\right) \in U_{a}$, then, by the statement containing equality (2.1), and the definition of $U_{a}$, we have

$$
\left|\left(f_{a} \diamond g_{a}\right)(z)\right|=\left|f_{a}\left(x^{\prime}\right) g_{a}\left(y^{\prime}\right)\right|>1-\varepsilon_{a},
$$

which means that $z$ lies in $V_{a}$, as claimed. On the other hand, since clearly $a$ lies in $U_{a}$, we can vary $a$ in $A$, and apply the compactness of $A$ to find $a_{1}, \ldots, a_{n} \in A$ such that $A \subseteq \bigcup_{i=1}^{n} U_{a_{i}}$. Then, invoking the claim, we derive $\varphi^{-1}(A) \subseteq \bigcup_{i=1}^{n} V_{a_{i}}$, and hence

$$
\left(\bigcap_{i=1}^{n} G_{a_{i}}\right) \cap \varphi^{-1}(A) \subseteq\left(\bigcap_{i=1}^{n} G_{a_{i}}\right) \cap\left(\bigcup_{i=1}^{n} V_{a_{i}}\right)=\emptyset .
$$

In this way $\bigcap_{i=1}^{n} G_{a_{i}}$ becomes a neighbourhood of $z_{0}$ in $Z$ contained in $Z \backslash \varphi^{-1}(A)$. Since $z_{0}$ is an arbitrary element of $Z \backslash \varphi^{-1}(A)$, we realize that $\varphi^{-1}(A)$ is closed in $Z$. Finally, since $A$ is an arbitrary closed subset of $X \times Y$ 
we conclude that $Z_{0}$ is closed in $Z$ (by noticing that $Z_{0}=\varphi^{-1}(X \times Y)$ ) and that $\varphi$ is continuous.

Taking in Theorem 2.1 the space $Y$ reduced to a point, we immediately get the following.

Corollary $2.2([3])$. Let $X, Z$ be compact Hausdorff topological spaces, and let $T: C(X) \rightarrow C(Z)$ be a linear isometry. Then there exist a closed subset $Z_{0}$ of $Z$, a continuous surjective mapping $\varphi: Z_{0} \rightarrow X$, and a normone element $\alpha \in C(Z)$ satisfying $|\alpha(z)|=1$ and

$$
T(f)(z)=\alpha(z) f(\varphi(z))
$$

for every $(z, f) \in Z_{0} \times C(X)$.

Corollary 2.3. For compact Hausdorff topological spaces X, Z, consider the following conditions:

(1) There exists a continuous surjective mapping from $Z$ to $X$.

(2) $C(X)$ is linearly isometric to a subspace of $C(Z)$.

(3) There exists a continuous surjective mapping from some closed subset of $Z$ to $X$.

Then $(1) \Rightarrow(2) \Rightarrow(3)$. Moreover, if $Z$ is metrizable, then $(2) \Leftrightarrow(3)$.

Proof. $(1) \Rightarrow(2)$. If there is a continuous surjective mapping $\theta: Z \rightarrow X$, then the mapping $h \mapsto h \circ \theta$ from $C(X)$ to $C(Z)$ is a linear isometry.

$(2) \Rightarrow(3)$. By Corollary 2.2.

If $Z_{0}$ is any metrizable closed subset of $Z$, then $C\left(Z_{0}\right)$ is linearly isometric to a subspace of $C(Z)$ (indeed, by the Borsuk-Kakutani theorem [2, Theorem 1.21], there is in fact a norm-one linear operator $S: C\left(Z_{0}\right) \rightarrow C(Z)$ satisfying $\left.S(f)\right|_{Z_{0}}=f$ for every $f \in C\left(Z_{0}\right)$ ). Now, assume that $Z$ is metrizable, and that there exists a continuous surjective mapping from a closed subset $Z_{0}$ of $Z$ to $X$. Then, since $C(X)$ is linearly isometric to a subspace of $C\left(Z_{0}\right)$ (by $(1) \Rightarrow(2)$ ), it follows that $C(X)$ is linearly isometric to a subspace of $C(Z)$.

REMARK 2.4. (a) Even if $Z$ is metrizable, the implication $(1) \Rightarrow(2)$ in Corollary 2.3 above is not reversible. Many counterexamples can be exhibited by keeping in mind the Banach-Mazur theorem that $C(X)$ is linearly isometric to $C(Z)$ whenever the compact spaces $X$ and $Z$ are metrizable and $Z$ is uncountable. Thus, with $X:=\{0,1\}$ and $Z:=[0,1]$, condition (2) in Corollary 2.3 is satisfied, whereas clearly condition (1) does not hold. In this case, an elementary embedding $C(X) \hookrightarrow C(Z)$ is the one assigning to each function from $\{0,1\}$ to $\mathbb{K}$ its unique affine extension to $[0,1]$.

(b) If $Z$ is not metrizable, the implication $(2) \Rightarrow(3)$ in Corollary 2.3 is not reversible either. Indeed, take $Z:=\beta(\mathbb{N})$ (the Stone-Cech compactification of the integers) and $X:=\beta(\mathbb{N}) \backslash \mathbb{N}$; then condition (3) is satisfied in an obvi- 
ous way, but condition (2) does not hold. Indeed, the norm of $C(Z)$ is determined by the family of all point evaluations on the set $\mathbb{N}$, whereas the norm of $C(X)$ cannot be determined by any countable subset of the closed unit ball of its dual (see the second paragraph after Proposition II.4.16 in [4]).

Corollary 2.5. For compact Hausdorff topological spaces $X, Y, Z$, consider the following conditions:

(1) There exists a continuous surjective mapping from $Z$ to $X \times Y$.

(2) $C(X \times Y)$ is linearly isometric to a subspace of $C(Z)$.

(3) There exists a bilinear mapping $\diamond: C(X) \times C(Y) \rightarrow C(Z)$ satisfying $\|f \diamond g\|=\|f\|\|g\|$ for every $(f, g) \in C(X) \times C(Y)$.

(4) There exists a continuous surjective mapping from some closed subset of $Z$ to $X \times Y$.

Then $(1) \Rightarrow(2) \Rightarrow(3) \Rightarrow(4)$. Moreover, if $Z$ is metrizable, then in fact we have $(2) \Leftrightarrow(3) \Leftrightarrow(4)$.

Proof. $(1) \Rightarrow(2)$. By Corollary 2.3 .

$(2) \Rightarrow(3)$. For $(f, g) \in C(X) \times C(Y)$, we define $f \otimes g \in C(X \times Y)$ by $(f \otimes g)(x, y):=f(x) g(y)$. If there exists a linear isometry

$$
\phi: C(X \times Y) \rightarrow C(Z),
$$

then the mapping $\diamond: C(X) \times C(Y) \rightarrow C(Z)$ defined by $f \diamond g:=\phi(f \otimes g)$ is bilinear and satisfies $\|f \diamond g\|=\|f\|\|g\|$ for every $(f, g) \in C(X) \times C(Y)$.

$(3) \Rightarrow(4)$. By Theorem 2.1.

In the case that $Z$ is metrizable, the implication $(4) \Rightarrow(2)$ follows from Corollary 2.3.

REMARK 2.6. We note that, when in Corollary 2.5 above we take $Y$ reduced to a point, then conditions (2) and (3) assert the same, and Corollary 2.5 becomes Corollary 2.3. Therefore, by Remark 2.4, neither of the implications $(1) \Rightarrow(2)$ (even if $Z$ is metrizable) and $(3) \Rightarrow(4)$ in Corollary 2.5 is reversible.

A more illuminating example that (4) does not imply (3) is the following. Take $Z:=\beta(\mathbb{N}) \times \beta(\mathbb{N})$ and $X=Y:=\beta(\mathbb{N}) \backslash \mathbb{N}$. Then, since $X \times Y$ is a closed subset of $Z$, condition (4) is satisfied in an obvious way. However, if condition (3) were satisfied, then, fixing a norm-one element $g$ of $C(Y)$, the mapping $f \mapsto f \diamond g$ from $C(X)$ to $C(Z)$ would be a linear isometry. But, since the norm of $C(Z)$ is determined by the countable family of all point evaluations on the set $\mathbb{N} \times \mathbb{N}$ (because the inclusion $\mathbb{N} \times \mathbb{N} \hookrightarrow \beta(\mathbb{N}) \times \beta(\mathbb{N})$ extends to a continuous surjective mapping from $\beta(\mathbb{N} \times \mathbb{N})$ to $\beta(\mathbb{N}) \times \beta(\mathbb{N})$ ), the existence of such an isometry is impossible (see Remark 2.4(b)).

Without the assumption of metrizability of $Z$, we do not know if the implication $(2) \Rightarrow(3)$ in Corollary 2.5 is reversible. 
3. Absolute-valuable $C(X)$-spaces. A Banach space $E$ is said to be absolute-valuable if there exists a bilinear mapping $\diamond: E \times E \rightarrow E$ satisfying $\|\xi \diamond \chi\|=\|\xi\|\|\chi\|$ for all $\xi, \chi \in E$. Let $X$ be a metrizable compact space. It follows from Corollary 2.5 that $C(X)$ is absolute-valuable if and only if there exists a continuous surjective mapping from some closed subset of $X$ to $X \times X$. In this section we will prove that in fact the absolute valuableness of $C(X)$ can be settled in terms of the cardinality of $X$. To this end, we need some elementary lemmas of pure topology. We feel that such lemmas are well known, but we give their proofs for the sake of completeness. As usual, for every topological space $X$, we define the derived set $X^{\prime}$ of $X$ as the set of all accumulation points of $X$.

LEMmA 3.1. Let $X$ and $Y$ be topological spaces, and let $\varphi: X \rightarrow Y$ be a continuous surjective mapping. Assume that $X$ is compact and that $Y$ is Hausdorff. Then $Y^{\prime} \subseteq \varphi\left(X^{\prime}\right)$.

Proof. For every point $z$ in a topological space, we denote by $\mathcal{V}(z)$ the set of all neighbourhoods of $z$. Let $y$ be in $Y^{\prime}$. Then, since $\varphi$ is surjective, for every $V \in \mathcal{V}(y)$ there exists $x_{V} \in X$ such that $\varphi\left(x_{V}\right) \in V \backslash\{y\}$. Considering in $\mathcal{V}(y)$ the order given by the inverse inclusion, the compactness of $X$ provides us with a cluster point $x \in X$ of the net $\left\{x_{V}\right\}_{V \in \mathcal{V}(y)}$. Since $\varphi$ is continuous, $\varphi(x)$ is a cluster point of the net $\left\{\varphi\left(x_{V}\right)\right\}_{V \in \mathcal{V}(y)}$. Since clearly $\left\{\varphi\left(x_{V}\right)\right\}_{V \in \mathcal{V}(y)}$ converges to $y$, and $Y$ is Hausdorff, it follows that $\varphi(x)=y$. Since $x$ is different from $x_{V}$ for every $V \in \mathcal{V}(y)$, and is a cluster point of the net $\left\{x_{V}\right\}_{V \in \mathcal{V}(y)}$, it lies in $X^{\prime}$.

LEMmA 3.2. Let $X$ and $Y$ be topological spaces, let $\varphi: X \rightarrow Y$ be a continuous mapping, and let $\left\{X_{\lambda}\right\}_{\lambda \in \Lambda}$ be a decreasing net of closed subsets of $X$. Assume that $X$ is compact and that $Y$ is Hausdorff. Then we have $\bigcap_{\lambda \in \Lambda} \varphi\left(X_{\lambda}\right)=\varphi\left(\bigcap_{\lambda \in \Lambda} X_{\lambda}\right)$.

Proof. Let $y$ be in $\bigcap_{\lambda \in \Lambda} \varphi\left(X_{\lambda}\right)$. Then for $\lambda \in \Lambda$ there exists $x_{\lambda} \in X_{\lambda}$ with $\varphi\left(x_{\lambda}\right)=y$. Taking a cluster point $x$ of the net $\left\{x_{\lambda}\right\}_{\lambda \in \Lambda}$ in $X$, and keeping in mind that $\left\{X_{\lambda}\right\}_{\lambda \in \Lambda}$ is a decreasing net of closed subsets of $X$, we deduce that $x$ belongs to $\bigcap_{\lambda \in \Lambda} X_{\lambda}$. Since $\varphi(x)=y$, it follows that $y$ lies in $\varphi\left(\bigcap_{\lambda \in \Lambda} X_{\lambda}\right)$.

Given a topological space $X$ and an ordinal $\alpha$, we apply transfinite induction to define the $\alpha$-derived set $X^{(\alpha)}$ of $X$ : we put $X^{(0)}:=X, X^{(\alpha+1)}:=$ $\left(X^{(\alpha)}\right)^{\prime}$, and $X^{(\alpha)}:=\bigcap_{\beta<\alpha} X^{(\beta)}$ when $\alpha$ is a limit ordinal.

LEMma 3.3. Let $X$ and $Y$ be topological spaces, let $\varphi: X \rightarrow Y$ be a continuous surjective mapping, and let $\alpha$ be an ordinal. Assume that $X$ is compact and that $Y$ is Hausdorff. Then $Y^{(\alpha)} \subseteq \varphi\left(X^{(\alpha)}\right)$.

Proof. We argue by transfinite induction on $\alpha$. The case $\alpha=0$ is clear. 
Assume that the inclusion $Y^{(\alpha)} \subseteq \varphi\left(X^{(\alpha)}\right)$ is true for some ordinal $\alpha$. Then, putting $Z:=\varphi^{-1}\left(Y^{(\alpha)}\right) \cap X^{(\alpha)}$ and $\psi:=\left.\varphi\right|_{Z}: Z \rightarrow Y^{(\alpha)}$, we can apply Lemma 3.1, with $\left(Z, Y^{(\alpha)}, \psi\right)$ instead of $(X, Y, \varphi)$, to derive that $Y^{(\alpha+1)} \subseteq$ $\varphi\left(Z^{\prime}\right)$. Since $Z \subseteq X^{(\alpha)}$, we obtain $Y^{(\alpha+1)} \subseteq \varphi\left(X^{(\alpha+1)}\right)$.

Now assume that $\alpha$ is a limit ordinal, and that the inclusion $Y^{(\beta)} \subseteq$ $\varphi\left(X^{(\beta)}\right)$ holds for every ordinal $\beta<\alpha$. Applying Lemma 3.2 we have $Y^{(\alpha)}=$ $\bigcap_{\beta<\alpha} Y^{(\beta)} \subseteq \bigcap_{\beta<\alpha} \varphi\left(X^{(\beta)}\right)=\varphi\left(\bigcap_{\beta<\alpha} X^{(\beta)}\right)=\varphi\left(X^{(\alpha)}\right)$.

Lemma 3.4. Let $X$ and $Y$ be topological spaces, and let $\alpha$ be an ordinal. Then $X^{(\alpha)} \times Y \subseteq(X \times Y)^{(\alpha)}$.

Proof. Straightforward by transfinite induction on $\alpha$.

We recall that a topological space $X$ is said to be scattered if for every nonempty closed subset $Y$ of $X$ we have $Y \backslash Y^{\prime} \neq \emptyset$.

TheOREM 3.5. For a compact Hausdorff topological space $X$, consider the following conditions:

(1) There exists a continuous surjective mapping from $X$ to $X \times X$.

(2) $C(X \times X)$ is linearly isometric to a subspace of $C(X)$.

(3) $C(X)$ is absolute-valuable.

(4) There exists a continuous surjective mapping from some closed subset of $X$ to $X \times X$.

(5) $X$ is either reduced to a point or nonscattered.

(6) $X$ is either reduced to a point or uncountable.

Then $(1) \Rightarrow(2) \Rightarrow(3) \Rightarrow(4) \Rightarrow(5) \Rightarrow(6)$. Moreover, if $X$ is metrizable, then $(2) \Leftrightarrow$ $(3) \Leftrightarrow(4) \Leftrightarrow(5) \Leftrightarrow(6)$.

Proof. $(1) \Rightarrow(2) \Rightarrow(3) \Rightarrow(4)$. By Corollary 2.5 .

$(4) \Rightarrow(5)$. Assume that $X$ is scattered, and that there exist a closed subset $X_{0}$ of $X$ and a continuous surjective mapping $\varphi: X_{0} \rightarrow X \times X$. Since $X$ is scattered and compact, there is an ordinal $\alpha$ such that $X^{(\alpha)}$ is finite and nonempty (see for example $[5,8.6 .8]$ ). Denote by $n$ and $m$ the cardinal numbers of $X^{(\alpha)}$ and $X$, respectively. Since $X^{(\alpha)} \times X \subseteq \varphi\left(X_{0}^{(\alpha)}\right)$ (by Lemmas 3.3 and 3.4), we have $n m \leq n$. This implies $m=1$.

$(5) \Rightarrow(6)$. This is because countable compact Hausdorff spaces are scattered.

If $X$ is uncountable and metrizable, then, by the Banach-Mazur theorem (see Remark 2.4(a)), $C(X \times X)$ is linearly isometric to a subspace of $C(X)$.

Remark 3.6. Even if $X$ is metrizable, the implication $(1) \Rightarrow(2)$ in Theorem 3.5 is not reversible. Indeed, for $X=[0,1] \cup\{2\}$, condition (2) is satisfied (by the Banach-Mazur theorem), whereas a connectedness argument shows that condition (1) does not hold. 
Without the assumption of metrizability of $X$, the implication $(5) \Rightarrow(6)$ is not reversible either. Indeed, if $X$ denotes the one-point compactification of an uncountable discrete space, then $X$ is scattered.

Without the assumption of metrizability of $X$, we do not know about the reversibility of any of the implications $(2) \Rightarrow(3) \Rightarrow(4) \Rightarrow(5)$.

Given an infinite set $\Gamma$, we denote by $c(\Gamma)$ the vector space over $\mathbb{K}$ of all functions from $\Gamma$ to $\mathbb{K}$ having a limit along the filter of all co-finite subsets of $\Gamma$, endowed with the sup norm. Since $c(\Gamma)$ is linearly isometric to the space of all $\mathbb{K}$-valued continuous functions on the scattered compact Hausdorff topological space which is the one-point compactification of the discrete space $\Gamma$, we derive from Theorem 3.5 the following.

Corollary 3.7 ([1]). Let $\Gamma$ be an infinite set. Then $c(\Gamma)$ is not absolute-valuable.

Acknowledgements. The authors are specially grateful to Y. Benyamini for deep suggestions allowing them to improve in an essential way an early version of the paper. They also thank J. Becerra and J. F. Mena for fruitful remarks.

\section{References}

[1] J. Becerra, A. Moreno, and Á. Rodríguez, Absolute-valuable Banach spaces, to appear.

[2] Y. Benyamini and J. Lindenstrauss, Geometric Nonlinear Functional Analysis, Vol. 1, Amer. Math. Soc. Colloq. Publ. 48, Amer. Math. Soc., 2000.

[3] W. Holsztyński, Continuous mappings induced by isometries of spaces of continuous functions, Studia Math. 26 (1966), 133-136.

[4] J. Lindenstrauss and L. Tzafriri, Classical Banach Spaces, Lecture Notes in Math. 338, Springer, Berlin, 1973.

[5] Z. Semadeni, Banach Spaces of Continuous Functions, Vol. I, Monograf. Mat. 55, PWN, Warszawa, 1971.

Departamento de Análisis Matemático

Facultad de Ciencias

Universidad de Granada

18071 Granada, Spain

E-mail: agalindo@ugr.es

apalacio@ugr.es 